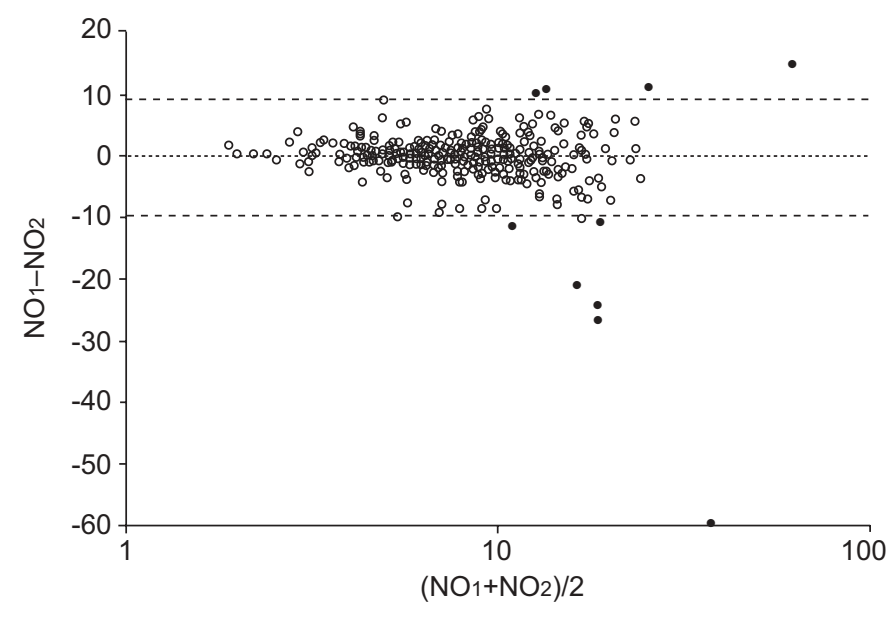

FIGURE 1. Bland Altman plot of the agreement between the duplicate fraction of exhaled nitric oxide ( $\mathrm{FeNO}$ ) measurements, indicating the mean difference between the duplicate FeNO measurements $(\cdots \cdots)$ and the mean difference $\pm 2 \mathrm{SD}$ (----). The open and solid circles indicate the children for whom the difference between duplicate $F$ eNO measurements was $<10 \mathrm{ppb}$ or $>10 \mathrm{ppb}$, respectively. The $x$-axis is logarithmic.

criteria were applied, the FeNO values were not more discriminative between children with and without asthma or atopy.

With respect to the ambient nitric oxide values, we have examined their effect on exhaled nitric oxide. Since there was no significant influence of ambient nitric oxide levels $<20 \mathrm{ppb}$ on the fraction of exhaled nitric oxide values in our study population (figs 2 and 3), we decided to include all children with ambient nitric oxide levels $<20 \mathrm{ppb}$ in the analyses. When the analyses were repeated, including only those children for whom the ambient nitric oxide values were $<10 \mathrm{ppb}$, similar results were observed.

J.E. Brussee* ${ }^{*}$ H.A. Smit* ${ }^{*}$ B. Brunekreef ${ }^{\#}$ and J.C. de Jongste ${ }^{\text {" }}$ ${ }^{*}$ Centre for Prevention and Health Services Research, National Institute for Public Health and the Environment, Bilthoven, \#Institute for Risk Assessment Sciences, Utrecht University, Utrecht and "Dept of Paediatrics, Division of Respiratory Medicine, Sophia's Children's Hospital, Erasmus University Medical Centre, Rotterdam, the Netherlands.

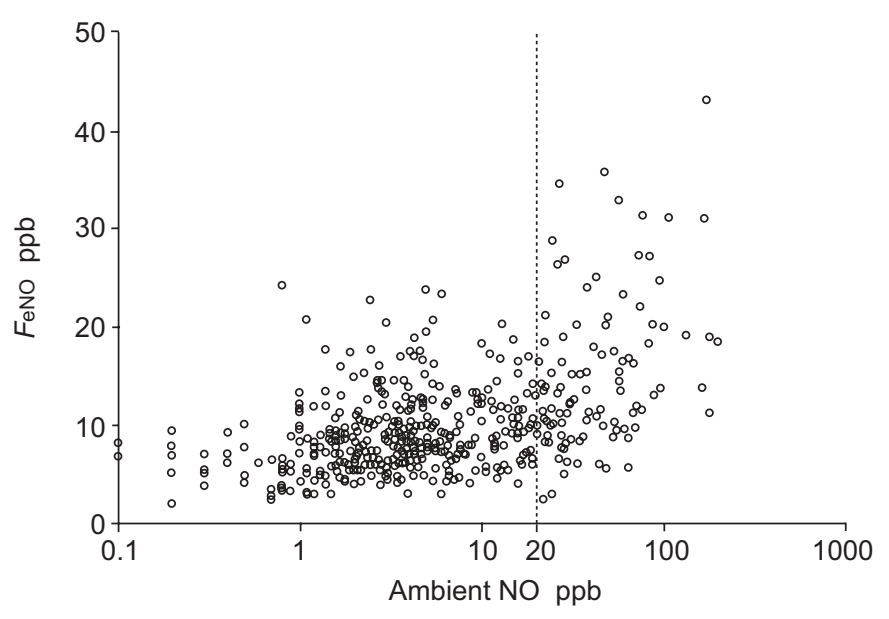

FIGURE 2. The influence of ambient nitric oxide (NO) levels on the fraction of exhaled nitric oxide $\left(F_{\mathrm{eNO}}\right)$ values in the study population. The $\mathrm{x}$-axis is logarithmic.

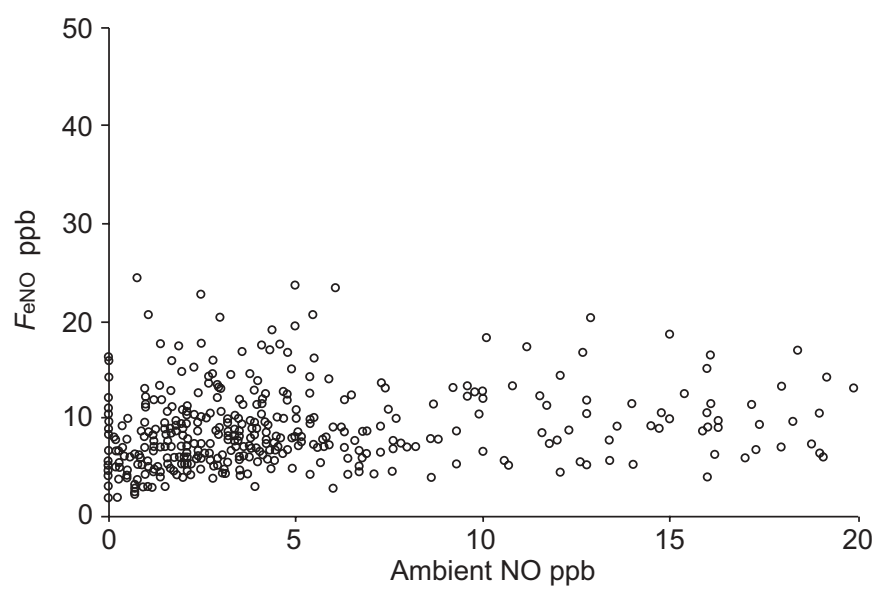

FIGURE 3. The influence of ambient nitric oxide (NO) levels $<20 \mathrm{ppb}$ on the fraction of exhaled nitric oxide $\left(F_{\mathrm{eNO}}\right)$ levels in the study population.

\section{REFERENCES}

1 Brussee JE, Smit HA, Kerkhof M, et al. Exhaled nitric oxide in 4-year-old children: relationship with asthma and atopy. Eur Respir J 2005; 25: 455-461.

DOI: $10.1183 / 09031936.05 .00050405$

\title{
Predictive value of BAL cellular analysis in differentiating pulmonary tuberculosis and sarcoidosis
}

\section{To the Editors:}

In a recent issue of the European Respiratory Journal, WELKER et al. [1] assessed the utility of bronchoalveolar lavage (BAL) cell counts and CD4/CD8 ratios as a test panel for the differential diagnosis of interstitial lung diseases (ILDs), and reported that their usage significantly modified the pre- versus post-test probability of a correct diagnosis. The diagnostic gain appeared particularly high in sarcoidosis, a disease where distinctive findings such as low BAL neutrophil counts, higher 
lymphocyte percentages and CD4/CD8 ratios, together with the high prevalence, increase the test panel predictive values [1].

In paucibacillary tuberculosis (TB), whose frequency is estimated between $29 \%$ and $80 \%$ of TB cases in Europe, BAL cytology is widely used, since confirmatory microbiology is lacking and different patterns of alveolar inflammation have been described in these two disorders [2-5]. Assessing the usefulness of BAL cellular profiles in differentiating sarcoidosis from TB may be of considerable clinical importance. In order to verify the reproducibility of the diagnostic tests described by WELKER et al. [1], we retrospectively analysed a series of consecutive patients undergoing pre-treatment BAL in a tertiary care centre (Carlo Forlanini Hospital, Rome, Italy) during a 4-yr period and in whom a final diagnosis of biopsyproven sarcoidosis or culture-positive pulmonary TB was established. The 88 sarcoidosis and 76 TB patients displayed significant differences (Mann-Whitney U-test) in BAL lymphocyte percentage (median (interquartile range) 30\% (16-49) in sarcoidosis versus $14 \%$ (4-29) in TB), neutrophil percentage (2\% (1-5) versus $3 \%(2-12))$ and CD4/CD8 ratio (3.9 (2.3-6.2) versus $2.2(1.2-3.8))$.

By applying the same cut-off used by WELKER et al. [1] to these three variables, it was found that the high-grade lymphocytosis $(>50 \%)$ was the best predictor. Since it was rarely observed in TB patients, as a consequence, its presence increased the probability of sarcoidosis from 0.54 (pre-test) to 0.91 ( $p=0.001$, Chi-squared test). The diagnostic gain was even higher if either a low $(<4 \%)$ neutrophil percentage or a high $(>3.5)$ CD4/CD8 ratio was associated with it (post-test probability $1, \mathrm{p}=0.001$, in both cases).

The value of a high CD4/CD8 ratio as an independent predictor of sarcoidosis was diminished by the scattered CD4/CD8 ratio distribution in TB patients: $29 \%$ of the TB patients evaluated had values of $>3.5(16 \%$ had values of $>5$ ). As a result, the pre-test probability of sarcoidosis only increased from 0.54 to 0.69 ( $p=0.049)$, a diagnostic gain lower than that observed by WELKER et al. [1] in their nonsarcoid ILD patient group. It is worth mentioning that very low $(<0.5)$ CD4/CD8 ratios (post-test probability 0.14, $\mathrm{p}=0.047$ ), particularly when combined with low lymphocyte percentages $(0.35, p=0.016)$, retained their ability to exclude sarcoidosis.

As normal values $(<4 \%)$ of neutrophil counts were found in half of TB patients, this criterion was unhelpful in differentiating sarcoidosis from TB. To the contrary, the presence of high-grade neutrophilic alveolitis $(>20 \%)$ rendered the diagnosis of sarcoidosis very unlikely (post-test probability 0.20, $\mathrm{p}=0.026)$.

Consistent with the data described by WELKER et al. [1], in our hands too, BAL cellular analysis provided additional information that either increased or decreased the probability of sarcoidosis with TB as the competing diagnosis. It should be stressed, however, that the proportion of sarcoidosis patients to which the predictive values apply is rather low: as a result of the poor sensitivity and specificity of the BAL parameters (55\% and $76 \%$, respectively, for high CD4/CD8 ratio, and $25 \%$ and $97 \%$, respectively, for high-grade lymphocytosis), only 48 had a "diagnostic" CD4/CD8 ratio and 22 a "diagnostic" lymphocytosis.

We also applied stepwise discriminant analysis for the diagnosis of sarcoidosis by using the leave-one-out crossvalidation method. While WELKER et al. [1] obtained a correct classification rate of $\sim 90 \%$ of sarcoidosis, $50 \%$ of hypersensitivity pneumonitis, $25 \%$ of usual interstitial pneumonia, $10 \%$ of bronchiolitis obliterans organising pneumonia and none of the nonspecific interstitial pneumonia patients, we found that the linear combination of the selected variables (CD4/CD8 ratio, lymphocyte and neutrophil percentages) correctly diagnosed $\sim 70 \%$ of our 164 patients either as sarcoid or tuberculosis. By considering the discriminant score as a single diagnostic test for sarcoidosis, sensitivity, specificity, positive and negative predictive values were $73 \%, 67 \%, 72 \%$ and $68 \%$, respectively. The use of cut-off values to classify variable and largely overlapping test results is expected to reduce the diagnostic power of the numeric data. Thus, although the use of cut-off values in the interpretation of bronchoalveolar lavage cellular results may appear more practical, the use of a discriminant score can provide clinicians with more accurate information guiding them through the diagnostic process, as previously suggested by DRENT et al. [6].

\section{S. Greco*, A. Marruchella\#, M. Massari ${ }^{\oplus}$ and C. Saltini ${ }^{+}$}

*Dept of Pulmonary Diseases, Azienda Ospedaliera San Camillo Forlanini, "Clinical Dept, INMI "L. Spallanzani", "National Centre for Epidemiology, Surveillance and Health Promotion, Istituto Superiore di Sanità, and ${ }^{+}$Dept of Internal Medicine, Università di Tor Vergata, Rome, Italy.

\section{REFERENCES}

1 Welker L, Jorres RA, Costabel U, Magnussen H. Predictive value of BAL cell differentials in the diagnosis of interstitial lung disease. Eur Respir J 2004; 24: 1000-1006.

2 WHO Collaborating Centre for the Surveillance of Tuberculosis in Europe. Surveillance of Tuberculosis in Europe - EuroTB. Report on tuberculosis cases notified in 2002. www.eurotb.org/rapports/2002/etb_tables_2002.pdf. Date last accessed: May 252005.

3 Anderson C, Inhaber N, Menzies D. Comparison of sputum induction with fiberoptic bronchoscopy in the diagnosis of tuberculosis. Am J Respir Crit Care Med 1995; 152: 1570-1574.

4 Hoheisel GB, Tabak L, Teschler H, Erkan F, Kroegel C, Costabel U. Bronchoalveolar lavage cytology and immunocytology in pulmonary tuberculosis. Am J Respir Crit Care Med 1994; 149: 460-463.

5 Drent M, Wagenaar SS, Mulder PH, van Velzen-Blad H, Diamant $\mathrm{M}$, van den Bosch JM. Bronchoalveolar lavage fluid profiles in sarcoidosis, tuberculosis, and nonHodgkin's and Hodgkin's disease. An evaluation of differences. Chest 1994; 105: 514-519.

6 Drent M, Mulder PG, Wagenaar SS, Hoogsteden HC, van Velzen-Blad H, van den Bosch JM. Differences in BAL fluid variables in interstitial lung diseases evaluated by discriminant analysis. Eur Respir J 1993; 6: 803-810.

DOI: $10.1183 / 09031936.05 .00042905$ 